\title{
Enhanced recovery pathways version 2.0 in thoracic surgery
}

\author{
Alessandro Brunelli ${ }^{1}$, Andrea Imperatori ${ }^{1}$, Andrea Droghetti ${ }^{2}$ \\ ${ }^{1}$ Department of Thoracic Surgery, St. James's University Hospital, Leeds, UK; ${ }^{2}$ Department of Thoracic Surgery, Carlo Poma Hospital, Mantova, Italy \\ Correspondence to: Dr. Alessandro Brunelli, MD. Department of Thoracic Surgery, St. James's University Hospital, Beckett Street, Leeds LS9 7TF, \\ UK. Email: brunellialex@gmail.com.
}

Submitted Oct 19, 2017. Accepted for publication Dec 11, 2017.

doi: $10.21037 /$ jtd.2017.12.81

View this article at: http://dx.doi.org/10.21037/jtd.2017.12.81

Enhanced recovery pathways (ERPs) after surgery is a multimodal plan of care based on the application of multiple standardized evidence-based elements with the aim to improve the perioperative patient experience and surgical outcomes. These standardized elements are not just limited to the postoperative period but refer to the preoperative phase and obviously the surgical procedure. ERP is based on the concept of "marginal gains", well known in sport. Applied in isolation, the individual elements may have not a great effect on the outcome, but when applied together they act synergistically (1).

The concept of ERP has been popularized in other specialties, particularly colorectal surgery, in which this practice has shown great benefits compared to standard care (2).

However, thoracic surgeons are used to the elements of ERP, even though these were not badged as such. Certain practices, such as fluid balance, pain management, early mobilization, postoperative rehabilitation, are used in many thoracic surgery centers since decades. Several years ago, Cerfolio and colleagues $(3,4)$ for instance identified modifiable and non-modifiable factors, which could contribute to improve fast track rate. Among the modifiable factors, the most relevant were the management of chest tubes, pain control, and social support plans. In spite of the factors contributing to ERP are well embedded in the thoracic surgery practice, the literature on ERP in our specialty is scant (5). Only few studies tried to analyze the effect of a standardized practice on the outcome after lung surgery. These studies were inconsistent in the elements they used to define a fast track protocol. The findings were also inconsistent. In general, the application of standardized elements of care contributed to reduce hospital stay. However, inconsistent findings were reported in terms of morbidity, mortality and re-admission rates (6-8).

In all specialties, minimally invasive surgery represents the most important element in an ERP program. Yet so far most of the studies in thoracic surgery failed to include patients submitted to video-assisted thoracoscopic surgery (VATS), mostly because they were conducted prior to the widespread use of this approach.

In a recent study, we were not able to find any difference in terms of morbidity, mortality and length of stay between patients submitted to VATS lobectomy before and after the introduction of a formal ERP program at our institution (9). The most likely explanation for this rather disappointing finding is that the majority of the key elements, which constitute ERP were already in place before the start of a formal ERP (i.e., use of a single drain, use of a digital drain system, similar pain management, postoperative rehabilitation, early oral feeding, etc.). The addition of few other elements such as the assumption of energy drinks before surgery to reduce the catabolic response or the use of warming blanket to prevent shivering and hypothermia among the others are unlikely to provide any benefits in a population who received most of the other ERP elements already although not in a formalized fashion. Finally, and probably most importantly, the study included only patients undergoing key-hole surgery. VATS is the key elements of ERP likely obscuring the effects of other elements when applied together with this approach.

Are we already in a post-ERP world? Are we thoracic surgeons so good that what our surgical colleagues of other specialties call "enhanced recovery" is for us "standard care" $(9,10)$. The practices of pain control, fluid restriction, early as possible mobilization, physiotherapy are all widely adopted by most of the thoracic surgery centers since decades.

In order to improve even more the experience with care 
of our patients we probably need to make a further step toward a higher level of perioperative care: an ERP version 2.0. Particularly in the face of an ever-increasing number of patients presenting with multiple co-morbidities and higher surgical risk and the advent of non-surgical lung cancer treatment modalities, we need to progress from a standardized, ready-made to a tailored surgical care modulated to the characteristics and preferences/values of the patients.

In this regard, the use of specific and prospective databases, like the one created by the Italian VATS Group, represent the essential element to identify and validate specific ERAS indicators for our specialty.

We need to move from the concept of outcomes to the one of values in healthcare (11). Value in healthcare is expressed by the equation quality over cost. Quality in lung cancer surgery is represented by longitudinal outcomes such as cancer specific survival and quality of life. When interpreted in this way, costs should not be limited to hospital costs, but include social costs. In other words, we need to shift our focus from myopic shortterm results toward a more patient centric evaluation of efficiency. This should be the benchmark to evaluate our performance, the effectiveness of new technologies or lung cancer treatments, and finally of any process of care aimed at improving patient-centered values. This is what we mean for ERP version 2.

\section{Acknowledgements}

None.

\section{Footnote}

Conflicts of Interest: The authors have no conflicts of interest to declare.

Cite this article as: Brunelli A, Imperatori A, Droghetti A. Enhanced recovery pathways version 2.0 in thoracic surgery. J Thorac Dis 2018;10(Suppl 4):S497-S498. doi: 10.21037/ jtd.2017.12.81

\section{References}

1. Kehlet H, Wilmore DW. Evidence-based surgical care and the evolution of fast-track. Ann Surg 2008;248:189-98.

2. Ljungqvist $\mathrm{O}$, Scott $M$, Fearon KC. Enhanced Recovery After Surgery: A Review. JAMA Surg 2017;152:292-8.

3. Cerfolio RJ, Pickens A, Bass C, et al. Fast-tracking pulmonary resections. J Thorac Cardiovasc Surg 2001;122:318-24.

4. Bryant AS, Cerfolio RJ. The influence of preoperative risk stratification on fast-tracking patients after pulmonary resection. Thorac Surg Clin 2008;18:113-8.

5. Fiore JF Jr, Bejjani J, Conrad K, et al. Systematic review of the influence of enhanced recovery pathways in elective lung resection. J Thorac Cardiovasc Surg 2016;151:708-15.e6.

6. Muehling BM, Halter GL, Schelzig H, et al. Reduction of postoperative pulmonary complications after lung surgery using a fast track clinical pathway. Eur J Cardiothorac Surg 2008;34:174-80.

7. Salati M, Brunelli A, Xiume F, et al. Does fast-tracking increase the readmission rate after pulmonary resection? A case-matched study. Eur J Cardiothorac Surg 2012;41:1083-7.

8. Numan RC, Klomp HM, Li W, et al. A clinical audit in a multidisciplinary care path for thoracic surgery: an instrument for continuous quality improvement. Lung Cancer 2012;78:270-5.

9. Brunelli A, Thomas C, Dinesh P, et al. Enhanced recovery pathway versus standard care in patients undergoing videoassisted thoracoscopic lobectomy. J Thorac Cardiovasc Surg 2017;154:2084-90.

10. Cerfolio RJ. Call it "fast tracking" or "enhanced recovery pathways"-No matter the name, it ain't nothing new to thoracic surgeons. J Thorac Cardiovasc Surg 2017;154:2091.

11. Porter ME. What is value in health care? $\mathrm{N}$ Engl J Med 2010;363:2477-81. 